\title{
Overexpression of Arabidopsis VIT1 Increases Accumulation of Iron in Cassava Roots and Stems
}

Narayanan Narayanan ${ }^{1 凶}$, Getu Beyene ${ }^{1}$, Raj Deepika Chauhan ${ }^{1}$, Eliana Gaitán-Solis ${ }^{1}$, Michael A. Grusak ${ }^{2}$, Nigel Taylor ${ }^{1}$, and Paul Anderson ${ }^{1}$

${ }^{1}$ Donald Danforth Plant Science Center, 975 N. Warson Road, St. Louis, MO 63132, USA

${ }^{2}$ USDA-ARS Children's Nutrition Research Center, Department of Pediatrics, Baylor College of Medicine, 1100 Bates Street, Houston, TX 77030, USA

${ }^{凶}$ Author for Correspondence:

Narayanan Narayanan

Donald Danforth Plant Science Center

975 North Warson Road

St. Louis, MO 63132 USA

Tel: $314-587-1254$

Fax: 314-587-1354

narayanan@danforthcenter.org 
Abstract

Iron is extremely abundant in the soil, but its uptake in plants is limited due to low solubility in neutral or alkaline soils. Plants can rely on rhizosphere acidification to increase iron solubility. AtVIT1 was previously found to be involved in mediating vacuolar sequestration of iron, which indicates a potential application for iron biofortification in crop plants. Here, we have overexpressed AtVIT1 in the starchy root crop cassava using a patatin promoter. Under greenhouse conditions, iron levels in mature cassava storage roots showed 3-4 times higher values when compared with wild-type plants. Significantly, the expression of AtVIT1 showed a positive correlation with the increase in iron concentration of storage roots. Conversely, young leaves of AtVIT1 transgenic plants exhibit characteristics of iron deficiency such as interveinal chlorosis of leaves (yellowing) and lower iron concentration when compared with the wild type plants. Interestingly, the AtVIT1 transgenic plants showed 4 and 16 times higher values of iron concentration in the young stem and stem base tissues, respectively. AtVIT1 transgenic plants also showed 2-4 times higher values of iron content when compared with wild-type plants, with altered partitioning of iron between source and sink tissues. These results demonstrate vacuolar iron sequestration as a viable transgenic strategy to biofortify crops and to help eliminate micronutrient malnutrition in at-risk human populations.

\begin{abstract}
Abbreviations
VIT1, Vacuolar Iron Transporter; YSL, Yellow Stripe Like; FRO2; Ferric Reductase Oxidase; IRT1, Iron-regulated transporter; FRD3, Ferric Reductase Defective ; OPT3, Oligo Peptide Transporter; At, Arabidopsis thaliana; EV, Empty vector; SD- Standard Deviation.
\end{abstract}

\title{
Keywords
}

Cassava, Iron, AtVIT1, source-sink, biofortification 


\section{Introduction}

Micronutrient malnutrition affects more than one-half of the world's population, and especially impairs health and wellbeing in women and preschool children [1]. Iron deficiency is one of the world's major nutritional problems resulting from prolonged negative iron balance due to inadequate dietary iron intake or absorption [2]. It was estimated that in 2011, 29\% (496 million) of non-pregnant women and 38\% (32.4 million) of pregnant women 15-49 years of age were anaemic [3]. The occurrence of anemia is greatest in South Asia, Central Africa, and West Africa [4]. Different strategies have been employed with the goal of preventing or minimizing the occurrence of iron deficiency anemia. These include iron supplementation through medicines, dietary intake from iron rich foods, fortification of processed foods and biofortification of staple food crops. Most of these strategies have limitations in developing countries where iron deficiency is predominant $[2,5]$. Both scientific evidence and predictive cost-benefit analyses show that biofortification is one of the most reliable and stable strategies to deliver elevated iron in the diet through staple food crops [6]. Biofortification can be achieved through conventional breeding or transgenic approaches [7-9]. Recent studies show that transgenic biofortification combined with cost-effective intervention strategies can be highly effective for addressing micronutrient malnutrition [10,11]. Conventional breeding relies on exploitation of natural variation of the target micronutrient within parental lines. Where such variation does not exist, metabolic engineering brings significant potential for enhancing micronutrient levels in crop plants $[12,13]$.

More than 800 million people worldwide rely on the starchy root crop cassava (Manihot esculenta Crantz) as a source of dietary calories [14]. Although an excellent source of starch, cassava storage roots provide insufficient iron, zinc, pro-vitamin A or vitamin E for a healthy diet, when considered in quantities normally consumed [14, 15]. Therefore, populations that rely on cassava, and which cannot regularly access meat, fish or vegetables, are at risk of micronutrient malnutrition. Efforts to enhance iron content of cassava storage roots through traditional breeding are constrained due to insufficient genetic variation for this trait within the existing germplasm [16]. Unfortunately, with a typical adult-sized cassava meal ( 500 g) providing only $30 \%$ of the minimum daily requirement of iron, a 3 - to 4 -fold increase in iron concentration would be needed to help one meet their daily iron requirement.

Biotechnological approaches that facilitate the integration of genes responsible for iron uptake and accumulation within plant organs are therefore attractive strategies for enhancing the nutritional value of crops such as cassava. Although iron is an abundant element in most soils, it is mostly present in its insoluble ferric form [17]. Plants have evolved two iron acquisition strategies. Strategy I plants, including the dicots (such as cassava) and non-graminaceous monocots, rely on a number of biochemical processes including: 1) acidification of the rhizosphere to solubilize ferric iron; 2) reduction of the solubilized Fe(III) by a membrane-bound $\mathrm{Fe}(\mathrm{III})$ chelate reductase at the root surface; 3) transport of the resulting $\mathrm{Fe}^{2+}$ into the plant root cells by a $\mathrm{Fe}^{2+}$ transporter, and for some species possibly 4) the secretion of flavins to also facilitate the solubilization of ferric iron. All four mechanisms are upregulated in roots under conditions of iron deficiency [18-21]. Strategy II plants are unique to graminaceous plants and employ a chelation-based mechanism involving the release of $\mathrm{Fe}(\mathrm{III})$-specific phytosiderophores (PS) and subsequent uptake of the Fe(III) phytosiderophore complexes via a specific transport system $[22,23]$. 

toxicity [24]. At the cellular level, iron can be incorporated into proteins or stored within cellular compartments. Ferritin is a major iron storage protein that can hold up to $4500 \mathrm{Fe}$ atoms per molecule in the chloroplast, and presumably mitochondria [25]. Vacuolar sequestration is also employed as a mechanism for regulating iron homeostasis with numerous genes identified that act to control iron trafficking between the cytosol and vacuole [26-30]. Of these, AtVIT1 (vacuolar iron transporter) has been shown to function specifically in the vacuolar transport of $\mathrm{Fe}$ into the xylem parenchyma of developing embryos in Arabidopsis thaliana [28]. While vit1-1 mutant seeds showed no change in their overall iron concentration compared with the wild type, altered distribution of iron in the seeds was observed [28]. The two orthologs of AtVIT1 in rice, OsVIT1 and OsVIT2, have been shown to catalyze vacuolar Fe transport [30]. Both genes can complement the yeast $\Delta c c c 1$ mutant and $\mathrm{Zn}$ transport mutant $\Delta z r c 1$, with vacuoles isolated from these cells showing increased $\mathrm{Fe}, \mathrm{Zn}$ and $\mathrm{Mn}$ concentration [30]. OsVIT1 and OsVIT2 are highly expressed in flag leaf blades and sheaths, and loss of function mutants have decreased iron concentration in flag leaves, suggesting a role for OsVIT1 and OsVIT2 in regulating iron partitioning between source and sink tissues [30]. Recently, three Arabidopsis proteins with homology to AtVIT1 called VTLs (vacuolar transporter -like) were identified and found to catalyze Fe transport into vacuoles and contribute to regulation of Fe homeostasis in planta [31].

Transgenic strategies are being explored as ways to increase iron content in crop plants. Recombinant expression of the soybean ferritin and Aspergillus niger phytase in maize resulted in a significant increase of total iron content in seeds [32]. Recently, iron biofortified rice was achieved by expressing the three transgenes ferritin, NAS and OSYSL. The resulting plants showed a six-fold increase in iron concentration in greenhouse-grown $\mathrm{T}_{2}$ polished seeds, and a 4.4-fold increase in field-grown $\mathrm{T}_{3}$ polished seeds compared to non-modified controls [33, 34]. Introducing rice and soybean ferritin into the endosperm of rice grain led to 3.4-fold increase in Fe concentration in the grain over the wild type plants [35]. Similar attempts to increase iron concentration in the edible tissues of root, tuber and fruit crops have been reported. Overexpression of soybean ferritin led to a 6.32-fold increase of iron in leaves of transgenic banana [36] and a 5.03-fold increase of iron in the leaves of transgenic pineapple [37]. However, in both cases no significant increase of iron in the edible tissues was achieved. Recently, the iron-specific assimilatory protein FEAl from Chlamydomonas reinhardtii was integrated into cassava and expressed under control of the storage organ specific type I patatin promoter. Regenerated plants showed an approximate three-fold increase in iron in their storage roots compared with the non-transgenic control plants when grown under greenhouse conditions [38]).

In the current study, to enhance the iron concentration of cassava storage roots, an approach involving vacuolar sequestration of iron was attempted by overexpressing a codon optimized Arabidopsis VIT1 under control of the type I patatin promoter. Compared with the wild type, transgenic plants showed 3-4 times higher values of iron concentration in storage roots as well as 4 and 16 times higher values of iron concentration in young stem and stem base tissues, respectively. Overexpression of AtVIT1 in cassava also altered iron content in different tissues, demonstrating altered partitioning between source and sink tissues. These results may indicate a 
potential strategy for iron biofortification of crop plants to improve human diet and decrease micronutrient malnutrition.

\section{Materials and Methods}

\subsection{Plant materials, growth conditions and genetic transformation}

Transgenic plants of cultivar TME 204 were produced via Agrobacterium-mediated transformation of friable embyogenic callus (FEC) as described by Chauhan et al [39]. Plants were established in the greenhouse and grown for up to 24 weeks for the production of shoots and storage roots. Plants were fertilized twice weekly at a rate of $100 \mathrm{ppm}$ with Jack's professional fertilizer (JR PETERS, INC, PA, USA) consisting of $10 \%$ nitrogen, $30 \%$ phosphate $\left(\mathrm{P}_{2} \mathrm{O}_{5}\right)$, and $20 \%$ soluble potash $\left(\mathrm{K}_{2} \mathrm{O}\right)$ along with $0.10 \%$ chelated iron [40]. At all other times plants were watered with reverse osmosis water 2-3 times per day as required.

\subsection{Total biomass and dry matter content}

Leaves and stems were harvested from 16-week-old greenhouse-grown plants and fresh weights were recorded. Storage and fibrous roots were cut from the stem tissues and soil was removed under running tap water. Tissue surfaces were dried with paper towels and fresh weights were recorded. The peel was removed from the storage roots, and chopped samples of the inner root were placed into $50 \mathrm{ml}$ falcon tubes, transferred to a $-80 \mathrm{C}$ freezer for 2-3 hours followed by freeze-drying using a lyophilizer (VirTis, PA) for at least 48 hours. Freeze dried samples were weighed to determine dry matter content. Total biomass per plant was calculated by adding all dry weight values of different tissues obtained from the same plant.

\subsection{Plasmid construction}

The construct p8012 was produced in which the T-DNA carried A. thaliana vacuolar iron transporter gene (AtVIT1;At2g01770[28]) driven by the type I patatin promoter from Solanum tuberosum (X03956, [41]) and its homologous 3' UTR The gene was synthesized (GenScript, Piscataway, NJ) after codon optimization and introduction of suitable restriction sites for subcloning. The expression cassette was assembled in an intermediate pBlueScript $(+)$ vector and moved to the pCAMBIA-2300-based binary vector for Agrobacterium-mediated transformation[39].

\subsection{RNA Extraction and cDNA Synthesis}

Total RNA was isolated from in vitro plants using the Promega SV-total RNA isolation kit (Madison, WI, USA) according to the manufacturer's instructions. Total RNA from greenhousegrown materials including young leaf (first formed leaf [0.3-0.5 g fresh weight $(\mathrm{FW})]$ ), mature leaf $\left(7^{\text {th }}\right.$ fully expanded leaf [0.7-1.0 g FW]), stems (5 nodes from base $\sim 10 \mathrm{~cm} \mathrm{[2-4} \mathrm{g} \mathrm{FW])} \mathrm{and}$ storage roots [12-20 g FW], was isolated using the CTAB- RNA extraction protocol [42]. Excessive DNA contamination was removed by treating with the TURBO DNA-free ${ }^{\mathrm{TM}}$ Kit (Ambion ${ }^{\circledR}$ by Life Technologies, Carlsbad, CA) according to the manufacturer's instructions and the concentration was assessed using a Nanodrop-2000C (Thermo-scientific, Wilmington, DE, USA). The structural integrity of the RNAs was checked on a non-denaturing agarose gel with ethidium bromide staining. Reactions were carried out with initial RNA template of 50-100 $\mathrm{ng} / \mu \mathrm{l}$ to a final volume of $20 \mu \mathrm{l}$. RNA samples were reverse transcribed with an anchored oligo $(\mathrm{dT})_{20}$ using Superscript ${ }^{\circledR}$ III- RT first strand cDNA synthesis system (Invitrogen, Carlsbad, CA, USA) in a volume of $20 \mu \mathrm{l}$ according to the manufacturer's instructions. 


\subsection{RT-PCR}

RT-PCR reactions were carried out using the gene specific primers AtVIT1-F: 5'CAGAGACAGAAGCTGCAGAGGTTG-3, and AtVIT1-R: 5, CGGATCTAAGAGGTTTGCTTCCAG -3'. Conditions for PCR were: $95^{\circ} \mathrm{C}$ for 2 min followed by $95^{\circ} \mathrm{C} 30 \mathrm{~s}, 56^{\circ} \mathrm{C} 30 \mathrm{~s}, 72^{\circ} \mathrm{C} 30 \mathrm{~s}$ for 30 cycles and a final extension at $72^{\circ} \mathrm{C}$ for $5 \mathrm{~min}$. Amplified products were visualized on a $1 \%$ TAE agarose gel containing ethidium bromide. Bands were photographed using the Molecular Imager Gel Doc ${ }^{\mathrm{TM}} \mathrm{XR}^{+}$Software System (BioRad, CA, USA).

\subsection{Real -Time PCR Analysis}

Real-time quantitative PCR (RT-qPCR) was performed using the Bio-Rad CFX Connect ${ }^{\mathrm{TM}}$ Real Time System (Bio-Rad, CA, USA) and Sso Advanced ${ }^{\mathrm{TM}}$ Universal SYBR ${ }^{\circledR}$ Green superMix (Bio-Rad, CA, USA) according to manufacturer's instructions. All primers (Table 1) were designed using Vector NTI Advance® 11.5 (Invitrogen). PCR cycling conditions comprised an initial denaturation holding stage at $95^{\circ}$ for $10 \mathrm{~min}$, followed by 40 cycles of cycling stage at 95 ${ }^{\circ} \mathrm{C}$ for $5 \mathrm{~s}, 59{ }^{\circ} \mathrm{C}$ for $30 \mathrm{~s}$ and final melt curve stage from $65^{\circ}$ to $95^{\circ}$ with 0.5 increments for $5 \mathrm{~s}$. For each sample, reactions were set up in quadruplicates and three biological plants per independent event were performed to ensure reproducibility of the results. Quantification of the relative transcript levels was performed using the comparative $\mathrm{C}_{\mathrm{T}}$ (threshold cycle) method [43]. Cytochrome c Oxidase $(C O X)$ and protein phosphatase 2A ( $p p 2 A)$ were used as housekeeping genes.[44]

\subsection{Cassava Database Search}

Blast search for iron metabolism genes was executed on draft genome sequence of database of cassava version 4.1 available publically at phytozome (http://www.phytozome.net/cassava.php/) [45]. Sequences from published Arabidopsis or rice metal regulated genes (GenBank or TAIR accession number indicated) including OsYSL1 (AB190912), AtFER (AF229850), AtIRT1 (AT4G19690), AtFRO2 (AT1G01580), AtFRD3 (NM_001161123.1), AtOPT3 (AT4G16370) were used as queries to search the annotated database of the cassava genome for putative homologs. Cassava accession numbers of the identified sequences used in the RT-qPCR are provided in Table 1.

\subsection{Perls' Prussian blue staining}

Cassava plants were sectioned freehand with a single-edged razor. Sections were placed in a 12 well plate and treated with Perls' Prussian blue stain (Sigma-Aldrich). Equal amounts of 4\% (v/v) $\mathrm{HCl}$ and $4 \%(\mathrm{w} / \mathrm{v})$ potassium ferrocyanide solutions were mixed immediately prior to use [46]. Sectioned materials were submerged in solution and incubated for 2-3 minutes at room temperature followed by rinsing in distilled water. Tissues were observed with the use of a Nikon C15304 microscope and images captured using Q-capture software.

\subsection{ICP analysis}

Tissues (60-100 mg DW) from greenhouse-grown transgenic and control plants were processed for mineral analysis. To remove extraplasmic Fe, roots were washed with millipore water for 3-4 minutes, followed by $5 \mathrm{mM}$ sodium dithionite and $1 \mathrm{M}$ magnesium sulphate for 4-5 minutes, and a 4-5 minute rinse with Milli-Q® water. Plant tissues were lyophilized for 48-72 h. Elemental 

DRC, West Melbourne, Australia) [47]. Peach leaf standards (SRM 1547A; National Institute of Standards and Technology, Gaithersburg, MD, USA) were digested and analyzed along with the experimental samples as an internal control to verify reliability of the procedures and analytical measurements. Four biological replicates and two technical replicates were processed for each sample.

\subsection{Statistical analysis}

Mineral content, concentration and agronomic data collected from four biological and two technical reps using material prepared from different tissues were subjected to one-way analysis of variance (ANOVA) and where significant, mean separation was done by Dunnett test using Minitab 17 Statistical Software [48]. For metal-regulated gene RT-QPCR studies, reactions were set up in quadruplicates using two biological replicates. Statistically significant differences between wild-type and transgenics were determined by student t-test.

\section{Results}

\subsection{Production and analysis of AtVIT1 transgenic cassava plants}

A total of 46 independent transgenic plants were generated by Agrobacterium-mediated transformation of cassava cultivar TME 204 using the construct p8012. PCR performed on genomic DNA of 40 of the 46 in vitro plant leaf samples showed presence of the AtVIT1 transgene (data not shown). The first 19 lines positive for the presence and expression of the AtVIT1 transgene (line numbers 8012-1 to 8012-19) (Fig. S1) were established and grown in the greenhouse for 16 weeks and used in this study. Within two weeks of transfer to soil, the youngest expanded leaves of 11 of the transgenic lines exhibited distinct interveinal chlorosis. An example of transgenic line 8012-17 is shown in Fig. 1B as compared to control plant (Fig. 1A). As individual leaves aged they became green, but the youngest expanded leaves of these transgenic lines consistently showed yellow coloration associated with iron deficiency throughout the 16-week observation period (Figs. 1D, F). The remaining eight lines showed normal leaf color in a manner identical to that seen in the non-transgenic wild type plants (Figs. $1 \mathrm{C}, \mathrm{E})$.

\subsection{AtVIT1 transgenic cassava plants showed altered iron concentration}

Iron concentration was determined by ICP-MS from the young leaf (first expanded leaf), mature leaf ( $7^{\text {th }}$ fully expanded leaf), young stem (uppermost 5 nodes), stem base (lowermost 5 nodes), storage root peel and storage root parenchyma at 16 weeks after planting. Determination of iron concentration in young leaves revealed 16 transgenic lines to have significantly reduced levels in this organ compared to the non-transgenic and the empty vector (EV) controls (Fig. 2A). Levels of iron in this young leaf were as low as $16.9 \mathrm{ppm}$, compared to wild type plants that averaged 56.4 ppm (Fig. 2A). Reduced levels of iron within young leaves of transgenic plants were positively correlated with presence of the yellow phenotype described above (Fig. 1), while transgenic lines 8012-3, 7 and 15 (which displayed a normal green phenotype in their young leaves) did not show significant reduction in iron concentration. Iron concentration in mature leaves ranged from $54 \mathrm{ppm}$ to $132 \mathrm{ppm}$ and was similar to control plants at $61.9 \mathrm{ppm}$ (Fig. 2B), with only transgenic line 8012-16 showing a significant increase in iron concentration for this organ compared with controls (Fig. 2B). 
Iron concentration was measured in stem and storage root sink tissues. While non-transgenic controls accumulated an average of $39.2 \mathrm{ppm}$ iron in young stem tissues, 8 transgenic lines accumulated significantly higher levels reaching a maximum of $155 \mathrm{ppm}$ in line 8012-16, an approximately 4 times higher value in iron concentration compared to control plants (Fig. 2C). A similar elevated pattern was observed for the stem base, with 14 lines showing a significant increase in iron concentration in transgenic lines compared with the non-transgenic and EV controls. Maximum concentration of 462 ppm was recorded in line 8012-16. This represents a value that is 16 times higher compared to $27-29 \mathrm{ppm}$ in non-transgenic control (Fig. 2D). Iron concentration within the storage roots was determined for both the peel and the root parenchyma tissues. No significant difference was seen in the peel iron concentration of transgenic lines compared to the wild type and EV controls (data not shown). In storage root parenchyma (edible) tissues, 15 of the 19 transgenic lines studied showed significantly elevated iron concentration (3-4 times higher value). Fifteen transgenic lines reached more than $20 \mathrm{ppm}$ in storage root tissues, with a maximum concentration of $48 \mathrm{ppm}$ recorded in line 8012-11. This represents a value that is 4 times higher compared to 11-12 ppm in non-transgenic control and EV plants (Fig. 2E).

Correlation of iron concentration between the various organs studied and that of the storage roots is shown in Fig. 3. A strong positive correlation was seen for iron concentration between the young stem $(r=0.55 ; p<0.001)$, stem base $(r=0.76 ; p<0.001)$ and storage roots, while a negative correlation ( $r=-0.73$ ) was obtained between the storage roots and young leaf (Fig. 3). All organ types described above were also analyzed for Cd (Fig. S2), Mn (Fig.S3), Zn (Fig. S4), Co (Fig. S5), Ni (Fig. S6) and Cu (Fig. S7) concentration in wild-type and transgenic plants. Manganese, zinc, cobalt and copper concentration showed a slight increase in all the tissues analyzed in the transgenic plants when compared with the control plants (Figs. S3-S5; S7). Nickel concentration in young leaves and mature leaves of transgenic plants showed a slight reduction when compared with the wild type plants (Fig S6-A, B).

\subsection{AtVIT1 transgenic plants show a differential spatial pattern of expression}

It was considered that variation in the initial color of young leaves, ranging from yellow to green, observed for some transgenic AtVITl plants could possibly be the result of differential expression of AtVIT1. To determine if this was the case, quantitative expression of the AtVIT1 transgene was analyzed by RT-qPCR in 15-week-old transgenic and wild type greenhousegrown plants. AtVIT1 expression at the RNA level was detected in young leaves, storage root tissues, stem base and fibrous roots. AtVIT1 expression was 10-20 times higher in storage roots than in young leaves (Fig. 4). A negative correlation was seen between iron concentration and expression of AtVIT1 in young leaves $(r=-0.54$; $p<0.01)$, with plants of transgenic lines 8012$2,-17$ and -12 (yellow colored young leaves) having lower iron concentration but higher expression of AtVIT1 in these tissues (Fig. 4A). Conversely, expression of AtVIT1 was positively correlated with iron concentration in the storage roots $(r=0.71 ; p<0.001)$. Transgenic plants of lines $8012-2,-17,-8$ and -12 which accumulated high levels of iron in the storage roots also showed high expression of AtVIT1 in this region (Fig. 4B). Other sink tissues including stem base and fibrous roots also showed higher transgene AtVIT1 expression (Fig. S8), correlating with the high iron concentration in these respective tissues (Fig. 2D).

\subsection{Total iron content of AtVITI transgenic cassava plants increased over time}


To better determine how iron concentration and total iron content in AtVITI transgenic plants increased, a time course experiment was performed for selected transgenic lines along with wild type and EV control. Transgenic lines 8012-1 (low iron accumulator), 8012-14 (medium iron accumulator), 8012-2, -5, -13 and -17 (high iron accumulators) were studied. Young leaf, mature leaves, petioles, young stem, stem base, fibrous roots and storage roots were sampled and analyzed for iron concentration at 12, 18 and 24 weeks after planting and dry weights were determined to allow calculation of total iron content. Only transgenic lines 8012-5 and -14 showed a significant increase in storage root iron concentration between 12 and 24 weeks in greenhouse conditions (Fig. 5A). Over the period of 12-24 weeks after planting, storage root iron content in wild type (from 115 to $259 \mu \mathrm{g}$ ) and empty vector control (from 111 to $252 \mu \mathrm{g}$ ) approximately doubled, while in most of the transgenic lines and especially in 8012-14, total iron content almost quadrupled (from 244 to $1085 \mu \mathrm{g}$ ) (Fig. 5B). In addition to the increase observed in storage roots, a 5-10 times higher value of iron content was observed in the stem base of transgenic lines compared with EV and wild type plants between 12 and 24 weeks (Fig. 5B). At all-time points studied, the higher accumulating transgenic lines exhibited total iron content that was 3-4 times higher value than control plants (Fig. 5B). For example, at 24 weeks, transgenic line 8012-17 had accumulated $4816 \mu \mathrm{g}$ of iron per plant compared with the wild type plants that averaged $1251 \mu \mathrm{g}$ per plant (Fig. 5B). Total iron content has been significantly altered at different tissue levels between the transgenic lines and the wild type plants. For example at 24 weeks, transgenic line 8012-17 showed a 2 times reduction of iron content in young leaves, 1.3 times higher value in other leaves, 3 times higher value in petiole, 2 times higher value in young stem, 8 times higher value in stem base, 5 times higher value in fibrous roots, and 4 times higher value in storage roots, when compared with the wild type plants (Fig. 5B).

\subsection{AtVIT1 transgenic cassava plants localize iron in and near the vascular tissues}

Iron localization within tissues and organs of transgenic plants was investigated using Perls' Prussian blue staining. Non-transgenic wild type plants showed very low-level signals in all the tissues including petiole, young stem, stem base, fibrous root and storage roots (Fig. 6). These wild type plants showed a blue staining associated with the vascular xylem in the stem and within the peel and cambium region of the storage root (Fig. 6). In contrast, distinct blue staining was observed in most of the AtVIT1 transgenic lines. One of the high iron accumulating transgenic lines, 8012-17, is shown as an example in Fig. 6. Petioles of transgenic lines showed high accumulation of iron within the vascular bundles (Fig. 6). Young stems showed significant accumulation of iron within the metaxylem of the young stem, and very strong staining within similar tissues of old stem sections. Fibrous roots were also seen to have accumulated significantly more iron than their non-transgenic counterparts. Iron localization within the storage roots was distinct and associated with cells close to the xylem vessels and tracheids within the parenchyma storage tissues (Fig. 6).

\subsection{Growth and phenotype of AtVIT1 transgenic cassava plants in greenhouse conditions} Plant biomass and dry matter were measured for all transgenic and wild type plants after 10 weeks growth under greenhouse conditions. No significant difference in total biomass and dry matter content of the storage roots was observed between the wild type and transgenic plants (Figs. S9A and B).

\subsection{Altered tissue-specific expression of metal-related genes in AtVIT1 expressing tissues}



RT-qPCR analysis across a suite of genes known to be involved in iron uptake and homeostasis. Genes assessed included: 1) Ferric Reductase Oxidase (FRO2); and 2) Iron-regulated transporter (IRT1), both of which play a major role in primary acquisition of iron from soil to the root; 3 ) Ferritin, which is involved in plastidic storage and iron detoxification; 4) Ferric Reductase Defective (FRD3), responsible for citrate efflux into the root vasculature; 5) Yellow Stripe-Like (YSL) of the family Fe (II)-nicotianamine transporters, proposed to function as key mediators of Fe uptake from the xylem, xylem unloading and intracellular transport in leaves and seeds; 6) Vacuolar Iron transporter (VIT1) for storing iron in vacuoles; and 7) Oligo Peptide Transporter (OPT3) which facilitates iron recirculation from the xylem to the phloem stream, and total iron redistribution from mature to developing tissues. The relative expression of these genes was determined in young leaf and storage roots of wild type controls and the high iron accumulating AtVIT1 transgenic line 8012-17. RT-qPCR results indicated that endogenous FRO2 was significantly upregulated in AtVIT1 expressing young leaves but downregulated in storage roots when compared with the wild type (Fig. 7A). IRT expression in transgenic young leaves showed a two-time reduction when compared with wild type but no significant difference was seen for expression of this gene in the storage roots (Fig. 7B). Both young leaves and storage roots showed a dramatic reduction of ferritin transcript levels in the transgenic plant (Fig. 7C). FRD3 and YSL expression showed a slight reduction in storage roots of transgenic line 8012-17 when compared with the wild type plants (Figs. 7D, 7E). No significant difference in endogenous VIT1 expression was seen in storage roots between the transgenic line and wild type plants (Fig. 7F), but VIT1 expression in young leaves was decreased more than two fold when compared with the control (Fig. 7F). Of the seven metal-associated genes examined, OPT3 expression was the most dramatically affected by overexpression of AtVIT1. OPT3 was seen to be highly upregulated in transgenic tissues showing a five-fold elevation in young leaves and 30-fold elevation in storage root tissues compared to the non-transgenic wild type plants (Fig. 7G).

\section{Discussion}

Biofortification of plants for human benefit has always been an important area of research. In this work, we overexpressed the Arabidopsis vacuolar iron transporter AtVIT1 in cassava leading to a 3-4 times higher value of iron concentration in the edible part of storage roots when compared with control plants. Movement of iron from soil to sink tissue involves the long distance transport, intercellular transport and the involvement of organelles [29, 49]. Among these organelles, the vacuole plays an important role in storing metals (both essential and toxic) as a "buffering pool" [49, 50]. Vacuolar sequestration as a strategy for increasing the iron content of staple food crops has not been widely explored. Recently in rice, disruption of OsVITI and OsVIT2 led to increased iron and zinc accumulation in seeds and a corresponding decrease in their concentration in the flag leaves[30], suggesting that iron and zinc translocation from source to sink, modulated by vacuolar storage capacity in the source organs, might represent a unique approach for biofortification.

Within two weeks of growth in the greenhouse, the youngest expanded leaves of transgenic AtVIT1 cassava lines exhibited interveinal chlorosis (Fig. 1) and had low iron concentration when compared with control plants, when measured at 17 weeks (Fig. 2A). This could have resulted, in part, from improper phloem-mediated redistribution of iron from mature leaves to sink tissues including the younger leaves [51-53]. Generally in plants, root-iron deficiency 

the leaves [54-56]. At the root level, two major transcriptional networks have been identified including the FIT and the POPEYE network [55-57], but the components of the systemic shootto-root Fe signaling pathway remain poorly understood. It is well known that phloem transport from old to new young leaves is more selective, and is mainly dependent on the phloem mobility [58]. However, to date, evidence is lacking about the transporters, ligands, and the chemical speciation of the putative phloem-mobile molecule mediating systemic Fe signaling.

In this study, transgenic lines showed a significant increase in iron concentration in different tissues including young stem (4 times higher value), stem base (16 times higher value) and storage root sink tissues (4 times higher value) relative to controls (Figs. 2C-E). These results are highly correlated with the increase of total iron content in the sink tissues of the transgenic plants (Fig. 5B). Since roots are considered as primary organs of iron absorption and they regulate iron transport to shoots $[54,59,60]$, it is likely that this iron in AtVIT1 transgenic plants is stored in the vacuoles and may account for this dramatic increase of the total iron determined in stem and storage roots. After uptake of iron from soil, it is still unclear which ligands bind iron, but buffering the iron uptake is very essential [29]. In our transgenic plants, the influx of iron may be buffered in the outer root layers which transport iron, presumably sequestering excess iron in the vacuole via VIT1. A similar buffering role has been proposed for IRT2, which transports iron and zinc [61] and has been localized to vesicles in the epidermis [62]. It is well known that iron moves symplastically through the interconnected cytoplasm of the root diffusing along the concentration gradient [63] and moves towards the shoot through the transpiration stream. In the AtVIT1 transgenic plants, iron might be effluxed into the xylem and actively localized in the xylem parenchyma (Fig. 6), sequestered into the vacuoles of these cells leading to a dramatic increase of iron in stem and storage root tissues (Fig. 2D, E). At the same time, the transpiration of iron to the youngest leaves might be reduced in AtVIT1 transgenic plants, due to less iron remaining in the xylem stream. This could explain the low iron concentration and chlorotic phenotype in the young leaves of AtVITl transgenic plants when compared with the wild type plants (Figs. 1D, 2A), as well as the weak negative correlation observed between the quantitative expression of AtVIT1 in young leaves of transgenic plants and leaf iron concentration (Fig. 4A). Apart from iron, other minerals such as manganese, zinc, cobalt and copper concentration showed a slight increase in all the tissues analyzed in the transgenic plants when compared with the control plants (Figs. S3-S5; S7) suggesting AtVIT1 might also transport these minerals.

In AtVIT1 transgenic plants, iron seems to be strongly localized within the vasculature in the sink tissues such as stem base and storage roots (Fig. 6), which correlates with both high iron concentration (Fig. 2D, E) and content (Fig. 5B) in the respective tissues when compared with the wild type control plants. It is probable that the Fe stored in the vacuoles of the vasculature may be in the $\mathrm{Fe}^{3+}$ form, as Perls' staining of iron in Arabidopsis embryos [64] strongly resembles the vascular localization of iron demonstrated by SXRF [28]. This suggests that VIT1 may be responsible for vacuolar Fe loading and the dramatic increase of the total iron determined in stem and storage roots. In addition, expression of AtVITl was positively correlated with iron concentration in the storage roots and fibrous roots (Fig. 4B, S8), demonstrating that there was adequate iron trafficked through these tissues to allow for increased sequestration. 

the transgenic cassava plants. Therefore, we studied the transcript accumulation of native endogenous metal regulated genes in tissues such as storage roots and young leaf. Our results showed that endogenous FRO2 was significantly upregulated in the young leaves of AtVIT1 transgenic plants when compared with the wild type suggesting that these tissues might be iron deficient (Fig. 7A). This also correlated with the yellow color and low iron concentration of the young leaves seen within these plants (Fig. 2A). In contrast, FRO2 expression was significantly lower in transgenic storage roots when compared with the control plants (Fig. 7A), suggesting that these tissues might be iron sufficient. This is consistent with the relatively high iron concentration in this organ (Fig. 2E). Both young leaves and storage roots showed a significant reduction of ferritin levels in the transgenic lines (Fig. 7C). For the transgenic leaves this seems reasonable, based on their low iron concentration. For the transgenic storage roots, this could imply that the iron levels are still too low to necessitate ferritin expression for iron storage, or that the absorbed iron is localized to vacuoles, consistent with the involvement of AtVIT1 [28, 65].

Other endogenous iron-related genes were investigated, including putative cassava homologs for FRD3, a YSL, and OPT3. The products of these genes are known to play roles in different aspects of whole-plant iron transport in the xylem or phloem pathways [66]. For instance, when iron enters the xylem, it binds with citrate [67] and in Arabidopsis citrate is effluxed into the xylem via FRD3, which is expressed in the root vasculature [46]. YSLs and OPTs are members of large gene families and their gene products are involved with the membrane transport of ironchelates or other metal chelates and include the transport of these species into the phloem pathway [66]. In this study, endogenous FRD3 and YSL expression showed a slight reduction in storage roots of transgenic line 8012-17 when compared with the wild type plants (Figs. 7D, 7E). The reason for this expression change is unclear to us, although they may be another reflection of the altered intracellular partitioning of iron that is probable in the AtVIT1 plants. OPT3 expression was highly upregulated in transgenic plants showing a five-fold elevation in young leaves and a 30 fold elevation in storage root tissues compared with wild type plants (Fig. 7F), suggesting that these plants might be attempting to recirculate iron from the xylem to the phloem stream. This hypothesis can be further studied by silencing the endogenous cassava OPT3 to see the effect of iron distribution in the plants.

Previously it was shown that transgenic expression of FEA1, from the unicellular algae Chlymadomonas reinhardtii, resulted in a threefold accumulation of iron in greenhouse-grown cassava storage roots [38]. FEA1 functions as an iron assimilatory protein and complements the Arabidopisis irtl mutant [68] and is thought to enhance iron uptake and transport from the soil. In contrast, evidence from A.thaliana [28] and rice [30] indicates that VIT1 operates by upregulating vacuolar sequestration and storage of iron. Additional work is required to confirm the mode of action of AtVIT1 in transgenic cassava. However, it can be hypothesized that upregulation of genes such as FEAl which enhance the uptake/transport of iron, in combination with genes that control sequestration/storage such as VIT1, could result in levels of iron biofortification greater than those reported here. We propose that the VIT1 vacuolar iron transporter has ideal characteristics for the iron biofortification of crops. The current study helps in highlighting the significance of vacuolar iron sequestration as a viable transgenic biofortification strategy for eliminating micronutrient malnutrition in humans. 


\section{Acknowledgements}

The authors thank Jackson Gehan, Paula Butts, May Wu, Adam Boyer, Valentina CarballoPortela, Amita Rai, Junying Ma, Tira Jones, Danielle Posey, and Jennifer Winch for their technical help and Patricia Cosgrove for formatting the references. The authors thank Dr. Ivan Baxter and Greg Zeigler, USDA Lab, DDPSC for all the ICP measurements in different tissues. The authors thank Dr. Mary Lou Guerinot, Dartmouth College for providing insights about the AtVIT1 gene and support during the study. This work was supported by the Bill \& Melinda Gates Foundation's Global Challenges for Global Health Program (Grant no: OPPGD1484) and in part by the US Department of Agriculture, Agricultural Research Service through Cooperative Agreement Number 58-6250-0-008 (to MAG). The contents of this publication do not necessarily reflect the views or policies of the US Department of Agriculture, nor does mention of trade names, commercial products, or organizations imply endorsement by the US Government. The Gates Foundation had no role in the study design; in the collection, analysis and interpretation of data; in the writing of the report; nor in the decision to submit the article for publication. 


\section{References}

[1] United Nations System, Standing Committee on Nutrition (SCN), 5th Report on the World Nutrition Situation. Nutrition for Improved Development Outcomes, WHO, Geneva, 2004.

[2] F.E. Viteri, Iron deficiency in children: new possibilities for its control, Intl. Child Hlth., 6 (1995) 49-62.

[3] World Health Organization, Global Nutrition Targets 2025: Anaemia policy brief WHO/NMH/NHD/14.4, WHO, Geneva, 2014.

[4] G.A. Stevens, M.M. Finucane, L.M. De-Regil, C.J. Paciorek, S.R. Flaxman, F. Branca, J.P. Pena-Rosas, Z.A. Bhutta, M. Ezzati, Global, regional, and national trends in haemoglobin concentration and prevalence of total and severe anaemia in children and pregnant and nonpregnant women for 1995-2011: a systematic analysis of population-representative data, Lancet Glob. Health, 1 (2013) e16-e25.

[5] E. Ridwan, W. Schultink, D. Dillon, R. Gross, Effects of weekly iron supplementation on pregnant Indonesian women are similar to those of daily supplementation, Am. J. Clin. Nutr., 63 (1996) 884-890.

[6] P. Nestel, H.E. Bouis, J.V. Meenakshi, W. Pfeiffer, Biofortification of staple food crops, J. Nutr., 136 (2006) 1064-1067.

[7] J.D. Haas, J.L. Beard, L.E. Murray-Kolb, A.M. Del Mundo, A. Felix, G.B. Gregorio, Ironbiofortified rice improves the iron stores of nonanemic Filipino women, J. Nutr., 135 (2005) 2823-2830.

[8] J. Gross, R.J. Stein, A.G. Fett-Netoi, J.P. Fett, Iron homeostasis related genes in rice, Genet. Mol. Biol., 26 (2003) 477-497.

[9] K.L. Hefferon, Nutritionally enhanced food crops; progress and perspectives, Int. J. Mol. Sci., 16 (2015) 3895-3914.

[10] M.T. Ruel, H. Alderman, Maternal, G. Child Nutrition Study, Nutrition-sensitive interventions and programmes: how can they help to accelerate progress in improving maternal and child nutrition? Lancet, 382 (2013) 536-551.

[11] H. De Steur, D. Blancquaert, S. Strobbe, W. Lambert, X. Gellynck, D. Van Der Straeten, Status and market potential of transgenic biofortified crops, Nat. Biotechnol., 33 (2015) 25-29. 
[12] L. Cong, C. Wang, L. Chen, H. Liu, G. Yang, G. He, Expression of phytoene synthase1 and carotene desaturase crtI genes result in an increase in the total carotenoids content in transgenic elite wheat (Triticum aestivum L.), J. Agric. Food Chem., 57 (2009) 8652-8660.

[13] R. Welsch, J. Arango, C. Bar, B. Salazar, S. Al-Babili, J. Beltran, P. Chavarriaga, H. Ceballos, J. Tohme, P. Beyer, Provitamin A accumulation in cassava (Manihot esculenta) roots driven by a single nucleotide polymorphism in a phytoene synthase gene, Plant Cell, 22 (2010) 3348-3356.

[14] R. Sayre, J.R. Beeching, E.B. Cahoon, C. Egesi, C. Fauquet, J. Fellman, M. Fregene, W. Gruissem, S. Mallowa, M. Manary, B. Maziya-Dixon, A. Mbanaso, D.P. Schachtman, D. Siritunga, N. Taylor, H. Vanderschuren, P. Zhang, The BioCassava Plus program: biofortification of cassava for sub-Saharan Africa, Annu. Rev. Plant Biol., 62 (2011) 251-272.

[15] M.B. Zimmermann, R.F. Hurrell, Improving iron, zinc and vitamin A nutrition through plant biotechnology, Curr. Opin. Biotechnol., 13 (2002) 142-145.

[16] A.L. Chavez, J.M. Bedoya, T. Sánchez, C. Iglesias, H. Ceballos, W. Roca, Iron, carotene, and ascorbic acid in cassava roots and leaves, Food Nutr. Bull., 21 (2000) 410-413.

[17] M.L. Guerinot, Y. Yi, Iron: Nutritious, noxious, and not readily available, Plant Physiol., 104 (1994) 815-820.

[18] D. Eide, M. Broderius, J. Fett, M.L. Guerinot, A novel iron-regulated metal transporter from plants identified by functional expression in yeast, Proc. Natl. Acad. Sci. U.S.A., 93 (1996) 5624-5628.

[19] J. Rodriguez-Celma, W. Schmidt, Reduction-based iron uptake revisited: on the role of secreted iron-binding compounds, Plant Signal Behav., 8 (2013) e26116.

[20] N.J. Robinson, C.M. Procter, E.L. Connolly, M.L. Guerinot, A ferric-chelate reductase for iron uptake from soils, Nature, 397 (1999) 694-697.

[21] G. Vert, N. Grotz, F. Dedaldechamp, F. Gaymard, M.L. Guerinot, J.F. Briat, C. Curie, IRT1, an Arabidopsis transporter essential for iron uptake from the soil and for plant growth, Plant Cell, 14 (2002) 1223-1233.

[22] C. Curie, Z. Panaviene, C. Loulergue, S.L. Dellaporta, J.F. Briat, E.L. Walker, Maize yellow stripe 1 encodes a membrane protein directly involved in Fe(III) uptake, Nature, 409 (2001) 346-349.

[23] L.A. Roberts, A.J. Pierson, Z. Panaviene, E.L. Walker, Yellow stripe1. Expanded roles for the maize iron-phytosiderophore transporter, Plant Physiol., 135 (2004) 112-120. 
[24] E.L. Connolly, M. Guerinot, Iron stress in plants, Genome Biol., 3 (2002) 1024.

[25] J.F. Briat, K. Ravet, N. Arnaud, C. Duc, J. Boucherez, B. Touraine, F. Cellier, F. Gaymard, New insights into ferritin synthesis and function highlight a link between iron homeostasis and oxidative stress in plants, Ann. Bot., 105 (2010) 811-822.

[26] S. Thomine, F. Lelievre, E. Debarbieux, J.I. Schroeder, H. Barbier-Brygoo, AtNRAMP3, a multispecific vacuolar metal transporter involved in plant responses to iron deficiency, Plant J., 34 (2003) 685-695.

[27] V. Lanquar, F. Lelievre, S. Bolte, C. Hames, C. Alcon, D. Neumann, G. Vansuyt, C. Curie, A. Schroder, U. Kramer, H. Barbier-Brygoo, S. Thomine, Mobilization of vacuolar iron by AtNRAMP3 and AtNRAMP4 is essential for seed germination on low iron, EMBO J., 24 (2005) 4041-4051.

[28] S.A. Kim, T. Punshon, A. Lanzirotti, L. Li, J.M. Alonso, J.R. Ecker, J. Kaplan, M.L. Guerinot, Localization of iron in Arabidopsis seed requires the vacuolar membrane transporter VIT1, Science, 314 (2006) 1295-1298.

[29] J. Morrissey, M.L. Guerinot, Iron uptake and transport in plants: the good, the bad, and the ionome, Chem. Rev., 109 (2009) 4553-4567.

[30] Y. Zhang, Y.H. Xu, H.Y. Yi, J.M. Gong, Vacuolar membrane transporters OsVIT1 and OsVIT2 modulate iron translocation between flag leaves and seeds in rice, Plant J., 72 (2012) 400-410.

[31] J. Gollhofer, R. Timofeev, P. Lan, W. Schmidt, T.J. Buckhout, Vacuolar-Iron-Transporter1like proteins mediate iron homeostasis in Arabidopsis, PLoS One, 9 (2014) e110468.

[32] G. Drakakaki, S. Marcel, R.P. Glahn, E.K. Lund, S. Pariagh, R. Fischer, P. Christou, E. Stoger, Endosperm-specific co-expression of recombinant soybean ferritin and Aspergillus phytase in maize results in significant increases in the levels of bioavailable iron, Plant Mol. Biol., 59 (2005) 869-880.

[33] H. Masuda, Y. Ishimaru, M.S. Aung, T. Kobayashi, Y. Kakei, M. Takahashi, K. Higuchi, H. Nakanishi, N.K. Nishizawa, Iron biofortification in rice by the introduction of multiple genes involved in iron nutrition, Sci. Rep., 2 (2012) 543.

[34] H. Masuda, T. Kobayashi, Y. Ishimaru, M. Takahashi, M.S. Aung, H. Nakanishi, S. Mori, N.K. Nishizawa, Iron-biofortification in rice by the introduction of three barley genes participated in mugineic acid biosynthesis with soybean ferritin gene, Front. Plant Sci., 4 (2013) 132. 
[35] N. Oliva, P. Chadha-Mohanty, S. Poletti, E. Abrigo, G. Atienza, L. Torrizo, R. Garcia, C. Duenas, Jr., M.A. Poncio, J. Balindong, M. Manzanilla, F. Montecillo, M. Zaidem, G. Barry, P. Herve, H. Shou, I.H. Slamet-Loedin, Large-scale production and evaluation of marker-free rice IR64 expressing phytoferritin genes, Mol. Breed., 33 (2014) 23-37.

[36] G.B. Kumar, L. Srinivas, T.R. Ganapathi, Iron fortification of banana by the expression of soybean ferritin, Biol. Trace Elem. Res., 142 (2011) 232-241.

[37] M. Mhatre, L. Srinivas, T.R. Ganapathi, Enhanced iron and zinc accumulation in genetically engineered pineapple plants using soybean ferritin gene, Biol. Trace Elem. Res., 144 (2011) 1219-1228.

[38] U.E. Ihemere, N.N. Narayanan, R.T. Sayre, Iron biofortification and homeostasis in transgenic cassava roots expressing the algal iron assimilatory gene, FEA1, Front. Plant Sci., 3 (2012) 171.

[39] R.D. Chauhan, G. Beyene, M. Kalyaeva, C.M. Fauquet, N. Taylor, Improvements in Agrobacterium-mediated transformation of cassava (Manihot esculenta Crantz) for large-scale production of transgenic plants, Plant Cell Tiss. Organ Cult., 121 (2015) 591-603.

[40] N. Taylor, E. Gaitan-Solis, T. Moll, B. Trauterman, T. Jones, A. Pranjal, C. Trembley, V. Abernathy, D. Corbin, C.M. Fauquet, A high-throughput platform for the production and analysis of transgenic cassava (Manihot esculenta) plants, Trop. Plant Biol., 5 (2012) 127-139.

[41] M. Bevan, R. Barker, A. Goldsbrough, M. Jarvis, T. Kavanagh, G. Iturriaga, The structure and transcription start site of a major potato tuber protein gene, Nucleic Acids Res., 14 (1986) $4625-4638$

[42] J.J. Doyle, J.L. Doyle, Isolation of plant DNA from fresh tissue, Focus, 12 (1990) 13-15.

[43] K.J. Livak, T.D. Schmittgen, Analysis of relative gene expression data using real-time quantitative PCR and the 2(-Delta Delta C(T)) Method, Methods, 25 (2001) 402-408.

[44] E. Ogwok, T. Alicai, M.E.C. Rey, G. Beyene, N.J. Taylor, Distribution and accumulation of cassava brown streak viruses within infected cassava (Manihot esculenta) plants, Plant Pathology, In Press (2015).

[45] S. Prochnik, P. Reddy Marri, B. Desany, P.D. Rabinowicz, C. Kodira, M. Mohiuddin, F. Rodriguez, C.M. Fauquet, J. Tohme, T. Harkins, D.S. Rokhsar, S. Rounsley, The Cassava Genome: current progress, future directions, Trop. Plant Biol., 5 (2012) 88-94.

[46] L.S. Green, E.E. Rogers, FRD3 controls iron localization in Arabidopsis, Plant Physiol., 136 (2004) 2523-2531. 
[47] G. Ziegler, A. Terauchi, A. Becker, P. Armstrong, K. Hudson, I. Baxter, Ionomic screening of field-grown soybean identifies mutants with altered seed elemental composition, Plant Genome, 6 (2013).

[48] Minitab 17 Statistical Software. [Computer software]. State College, PA: Minitab, Inc. (http://www.minitab.com), (2010).

[49] J.S. Peng, J.M. Gong, Vacuolar sequestration capacity and long-distance metal transport in plants, Front. Plant Sci., 5 (2014) 19.

[50] D.G. Mendoza-Cozatl, Q. Xie, G.Z. Akmakjian, T.O. Jobe, A. Patel, M.G. Stacey, L. Song, D.W. Demoin, S.S. Jurisson, G. Stacey, J.I. Schroeder, OPT3 is a component of the ironsignaling network between leaves and roots and misregulation of OPT3 leads to an overaccumulation of cadmium in seeds, Mol. Plant, 7 (2014) 1455-1469.

[51] D.G. Mendoza-Cozatl, T.O. Jobe, F. Hauser, J.I. Schroeder, Long-distance transport, vacuolar sequestration, tolerance, and transcriptional responses induced by cadmium and arsenic, Curr. Opin. Plant Biol., 14 (2011) 554-562.

[52] C.M. Palmer, M.L. Guerinot, Facing the challenges of $\mathrm{Cu}, \mathrm{Fe}$ and $\mathrm{Zn}$ homeostasis in plants, Nat. Chem. Biol., 5 (2009) 333-340.

[53] N. Verbruggen, C. Hermans, H. Schat, Molecular mechanisms of metal hyperaccumulation in plants, New Phytol., 181 (2009) 759-776.

[54] G.A. Vert, J.F. Briat, C. Curie, Dual regulation of the Arabidopsis high-affinity root iron uptake system by local and long-distance signals, Plant Physiol., 132 (2003) 796-804.

[55] E.L. Walker, E.L. Connolly, Time to pump iron: iron-deficiency-signaling mechanisms of higher plants, Curr. Opin. Plant Biol., 11 (2008) 530-535.

[56] M.N. Hindt, M.L. Guerinot, Getting a sense for signals: regulation of the plant iron deficiency response, Biochim. Biophys. Acta, 1823 (2012) 1521-1530.

[57] T.A. Long, H. Tsukagoshi, W. Busch, B. Lahner, D.E. Salt, P.N. Benfey, The bHLH transcription factor POPEYE regulates response to iron deficiency in Arabidopsis roots, Plant Cell, 22 (2010) 2219-2236.

[58] V. Fernández, T. Sotiropoulos, P. Brown, Foliar Fertilization: Scientific Principles and Field Practices, IFA, Paris, 2013.

[59] H.F. Bienfait, W. Van Den Briel, N.T. Mesland-Mul, Free space iron pools in roots: generation and mobilization, Plant Physiol., 78 (1985) 596-600. 
[60] S. Legay, C. Guignard, J. Ziebel, D. Evers, Iron uptake and homeostasis related genes in potato cultivated in vitro under iron deficiency and overload, Plant Physiol. Biochem., 60 (2012) 180-189.

[61] G. Vert, J.F. Briat, C. Curie, Arabidopsis IRT2 gene encodes a root-periphery iron transporter, Plant J., 26 (2001) 181-189.

[62] G. Vert, M. Barberon, E. Zelazny, M. Seguela, J.F. Briat, C. Curie, Arabidopsis IRT2 cooperates with the high-affinity iron uptake system to maintain iron homeostasis in root epidermal cells, Planta, 229 (2009) 1171-1179.

[63] H. Marschner, Mineral Nutrition of Higher Plants, 2nd ed., Academic Press, Boston, 1995.

[64] M.G. Stacey, A. Patel, W.E. Mcclain, M. Mathieu, M. Remley, E.E. Rogers, W. Gassmann, D.G. Blevins, G. Stacey, The Arabidopsis AtOPT3 protein functions in metal homeostasis and movement of iron to developing seeds, Plant Physiol., 146 (2008) 589-601.

[65] H. Roschzttardtz, G. Conejero, C. Curie, S. Mari, Identification of the endodermal vacuole as the iron storage compartment in the Arabidopsis embryo, Plant Physiol., 151 (2009) 13291338 .

[66] C. Curie, G. Cassin, D. Couch, F. Divol, K. Higuchi, M. Le Jean, J. Misson, A. Schikora, P. Czernic, S. Mari, Metal movement within the plant: contribution of nicotianamine and yellow stripe 1-like transporters, Ann. Bot., 103 (2009) 1-11.

[67] R. Rellan-Alvarez, J. Giner-Martinez-Sierra, J. Orduna, I. Orera, J.A. Rodriguez-Castrillon, J.I. Garcia-Alonso, J. Abadia, A. Alvarez-Fernandez, Identification of a tri-iron(III), tri-citrate complex in the xylem sap of iron-deficient tomato resupplied with iron: new insights into plant iron long-distance transport, Plant Cell Physiol., 51 (2010) 91-102.

[68] N.N. Narayanan, U. Ihemere, W.T. Chiu, D. Siritunga, S. Rajamani, S. Singh, S. Oda, R.T. Sayre, The iron assimilatory protein, FEA1, from Chlamydomonas reinhardtii facilitates ironspecific metal uptake in yeast and plants, Front. Plant Sci., 2 (2011) 67. 


\section{Figure Legends}

Figure 1: Phenotype of AtVIT1 transgenic cassava plant (8012-17) showing yellow coloration in young leaf. Two-week-old plants in Greenhouse (A, B); 7-week-old plants in greenhouse $(\mathrm{C}, \mathrm{D})$; 12-week-old plants in greenhouse $(\mathrm{E}, \mathrm{F})$.

Figure 2: Iron concentration of 16-week-old greenhouse-grown AtVIT1 transgenic and wild type cassava plants. Different tissues as shown were collected and subjected to ICP elemental analysis. Values are average of four biological reps and two technical reps. Error bars represent SD. EV: Empty Vector control. * and $* *$ denote significant differences at $p<0.05$ and $p<0.01$, respectively.

Figure 3: Correlation of iron concentration with different tissues of AtVIT1 transgenic plants. Negative correlation between the storage roots and young leaf (A) positive correlation between the storage roots and old leaf (B), young stem (C) and stem base (D). *** denotes significant differences at $p<0.001$.

Figure 4: Quantitative expression of AtVIT1 in transgenic plants. Young leaf (A) and storage roots (B) were collected from 15-week-old plants from greenhouse. Expression was compared and normalized to Cytochrome Oxidase gene (COX). Line 8012-1 expression values were adjusted to a value of 1 and all other expression values were normalized relative to this tissue. For each sample, reactions were set up in quadruplicates using two biological replicates. The black line above the white bars indicates the concentration of iron in respective tissues showing a negative correlation for young leaf (A) and positive correlation for storage roots (B). Error bars represent SD. $* *$ and $* * *$ denote significant differences at $p<0.01$ and $p<0.001$, respectively.

Figure 5: Iron Concentration and Total Iron Content of AtVIT1 transgenic plants under greenhouse conditions. A, Storage roots at different time points were collected and subjected to ICP elemental analysis. Iron concentration was measured in storage roots and shown as labeled. EV: Empty vector. B, Total iron content of different tissues collected at 12, 18 and 24 weeks. $*$ denotes significant differences at $p<0.05$.

Figure 6: Iron localization in AtVIT1 transgenic plant (8012-17). Tissues from 8- to 9-weekold plants were sectioned and stained with Perl's stain to visualize iron localization. Pictures were taken using a dissection microscope.

Figure 7: Differential expression of metal regulated genes in AtVIT1 transgenic cassava. Relative expression of metal regulated genes as indicated in wild type and AtVITI transgenic line (8012-17). Tissues (young leaf and storage roots) were collected from 16-week-old greenhouse plants. Expression of the metal regulated genes was normalized to $p p 2 A$. Wild-type root expression levels were adjusted to a value of 1 and all other expression values were normalized relative to this tissue. For each sample, reactions were set up in quadruplicates using two biological replicates. Error bars represent SD. The asterisk $(*)$ indicates statistically significant differences between wild type and transgenics, determined by student t-test with $\mathrm{p}<0.05$. 
Table 1: Forward and reverse specific primers for quantitative PCR (real-time) amplification are presented along with their cassava accession numbers.

\begin{tabular}{|l|l|l|l|}
\hline \multicolumn{1}{|c|}{ Gene } & \multicolumn{1}{c|}{ Cassava Accession } & \multicolumn{1}{c|}{ Forward primer } & \multicolumn{1}{c|}{ Reverse Primer } \\
\hline$M e F R O$ & cassava4.1_002675m & CTGGTCCCAAGAAGATGAGACATG & GCACTTTCACGCGTAGCATACA \\
\hline$M e I R T 1$ & cassava4.1_010600m & GCCTCTCATGGGCATGCTCA & TGAGTGCTGCTACAAGGGGC \\
\hline$M e F E R$ & cassava4.1_013978m & TAAGGATGGTGGGGAAAGGC & AAGCTCCCAATGAAGAAGAGTCCT \\
\hline$M e F R D 3$ & cassava4.1_006157m & TTGACGGAGTCAACTTTGGAGC & ACTAGTACATCCTGATAAACTTCCAGGGTCC \\
\hline$M e Y S L$ & cassava4.1_002751m & CCCTAGCAGGGATCAAACCACC & CCGTCTAGACAAGAATTGCCCC \\
\hline$M e V I T 1$ & cassava4.1_014806m & GTTGCGTTGCTGATATTTGGCT & TTACCGGAAGGATTATGCATGG \\
\hline$M e O P T 3$ & cassava4.1_002314m & GCACCTGGTATCGCAGTTAAAGGA & GCACTTGAAGAGAATGACCATCGA \\
\hline$P P 2 \mathrm{~A}$ & & TGCAAGGCTCACACTTTCATC & CTGAGCGTAAAGCAGGGAAG \\
\hline
\end{tabular}


FIGURES
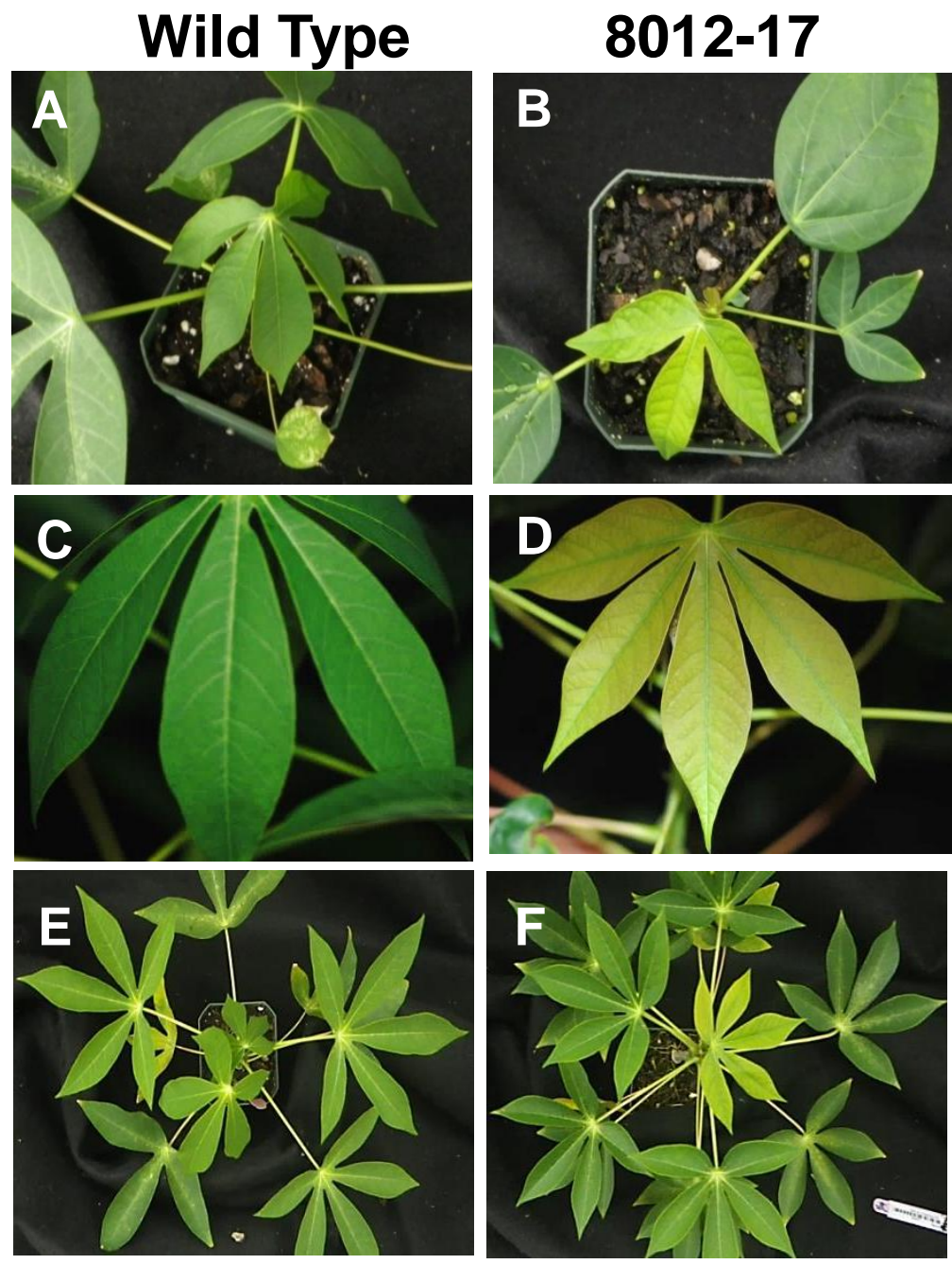

Figure 1 

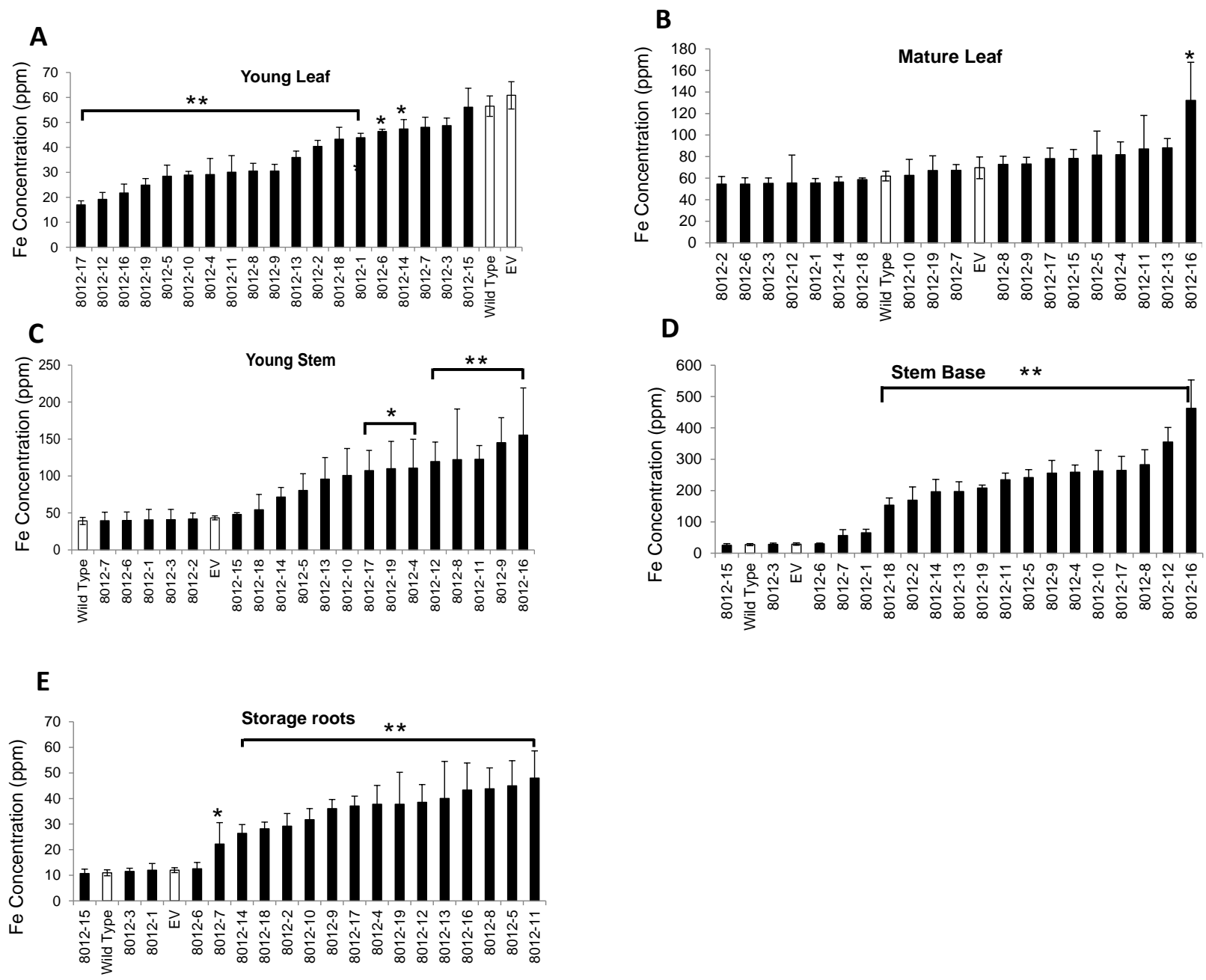

Figure 2 

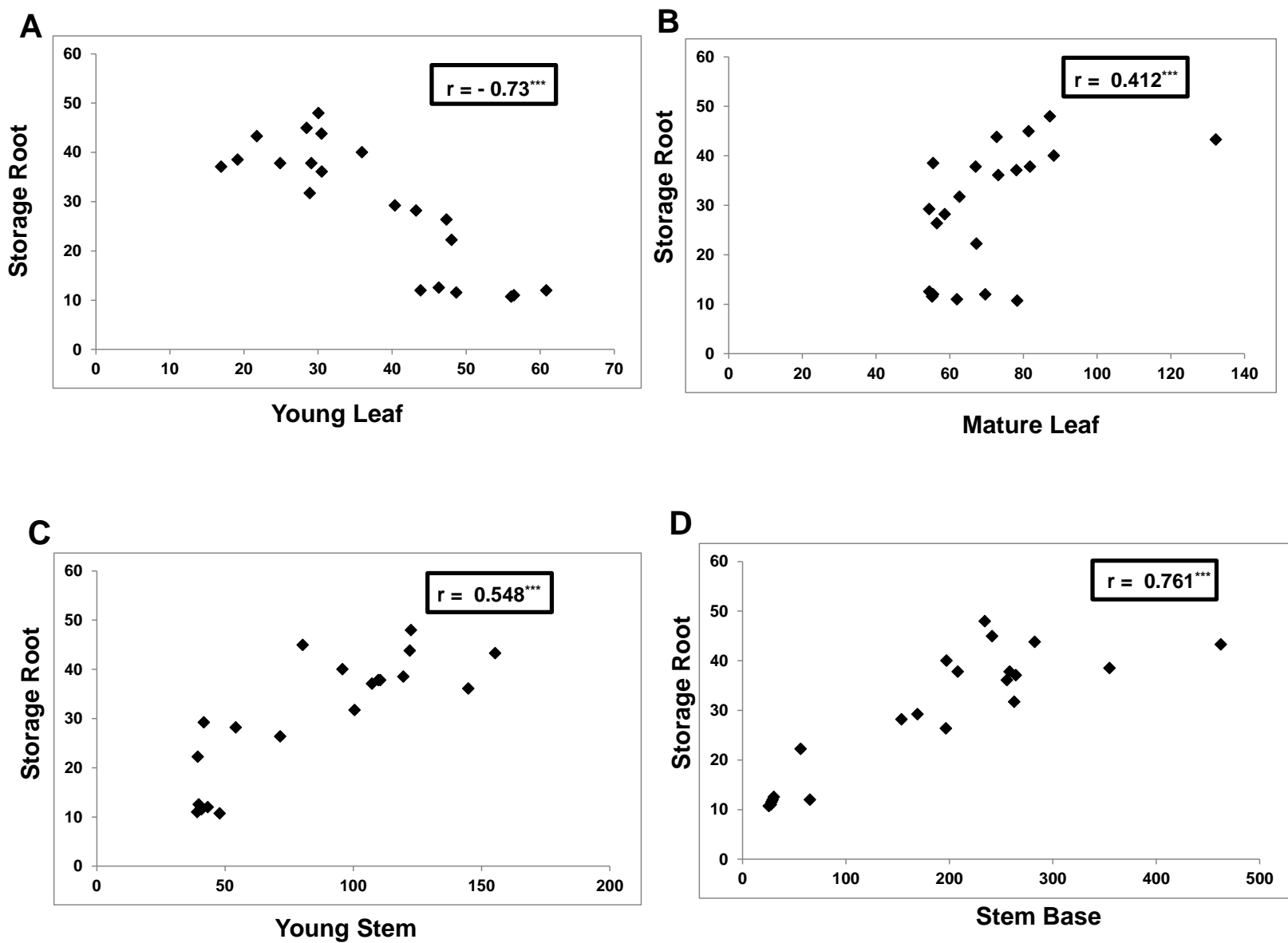

Figure 3 

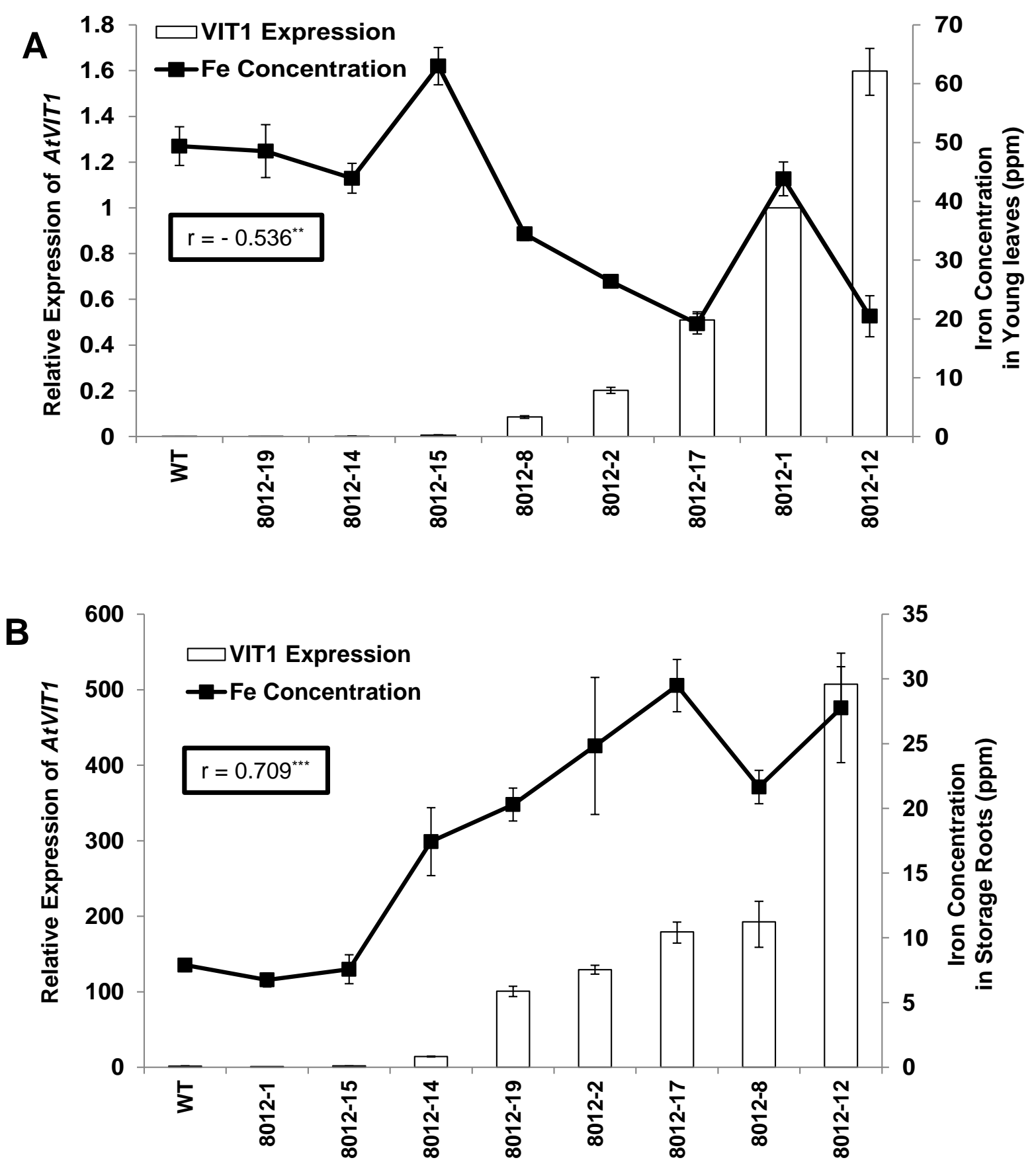

Figure 4 

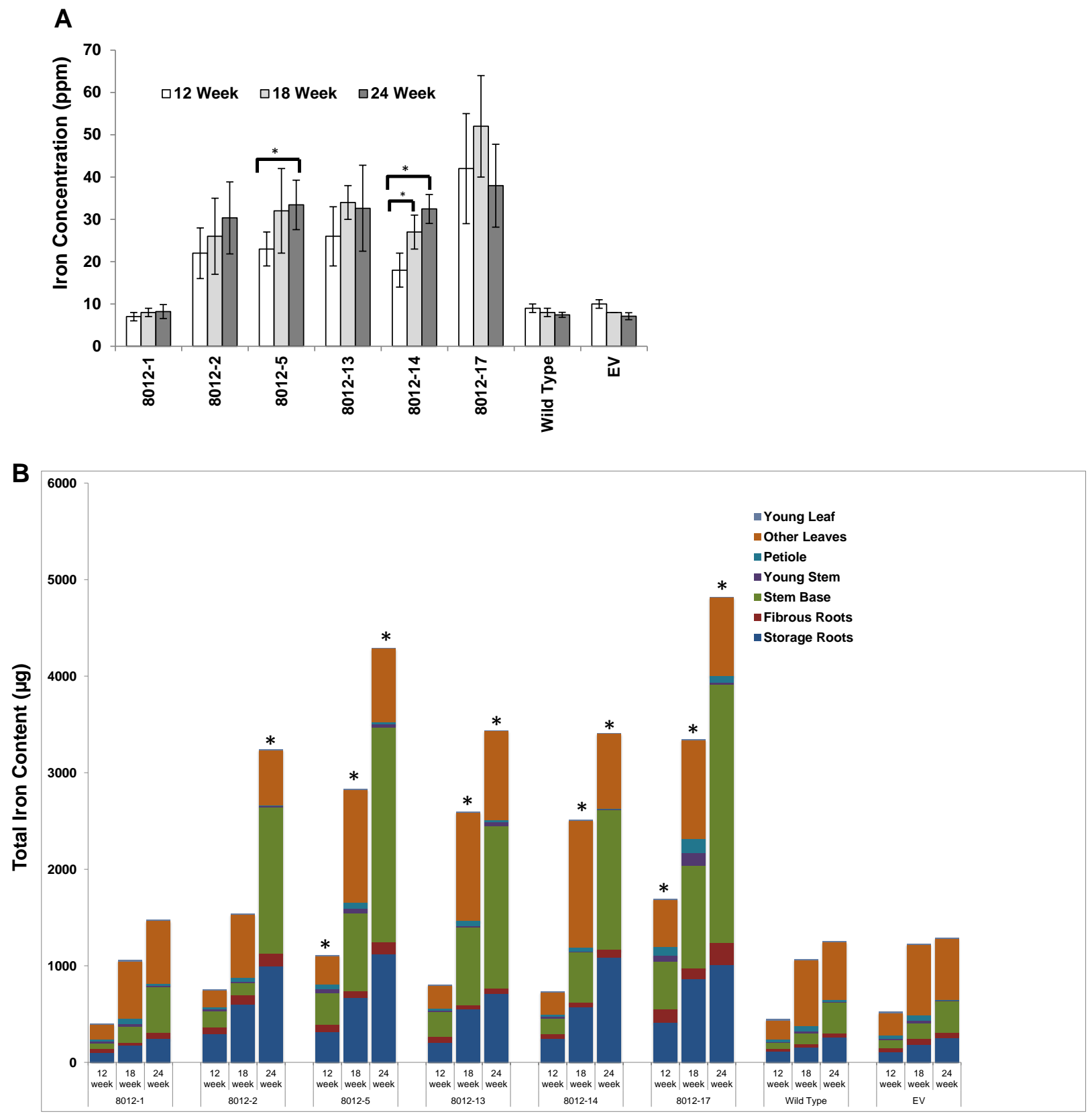

Figure 5. 


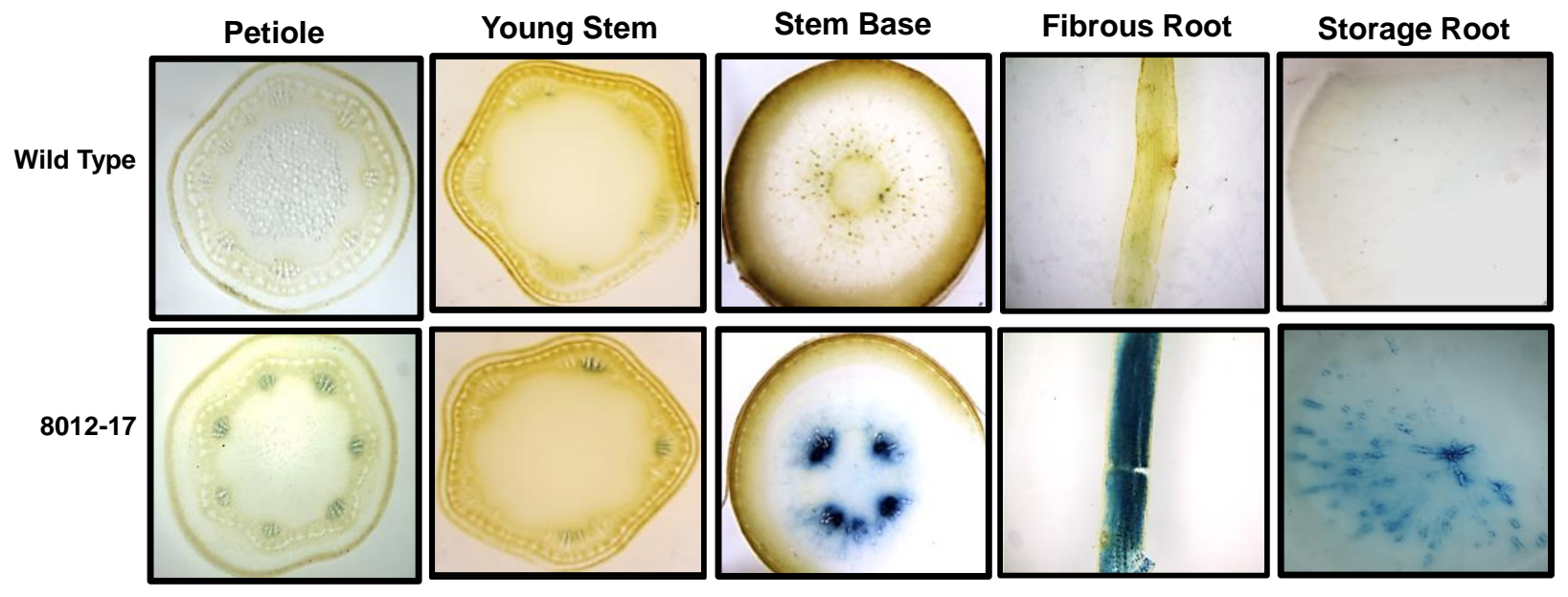

Figure 6 

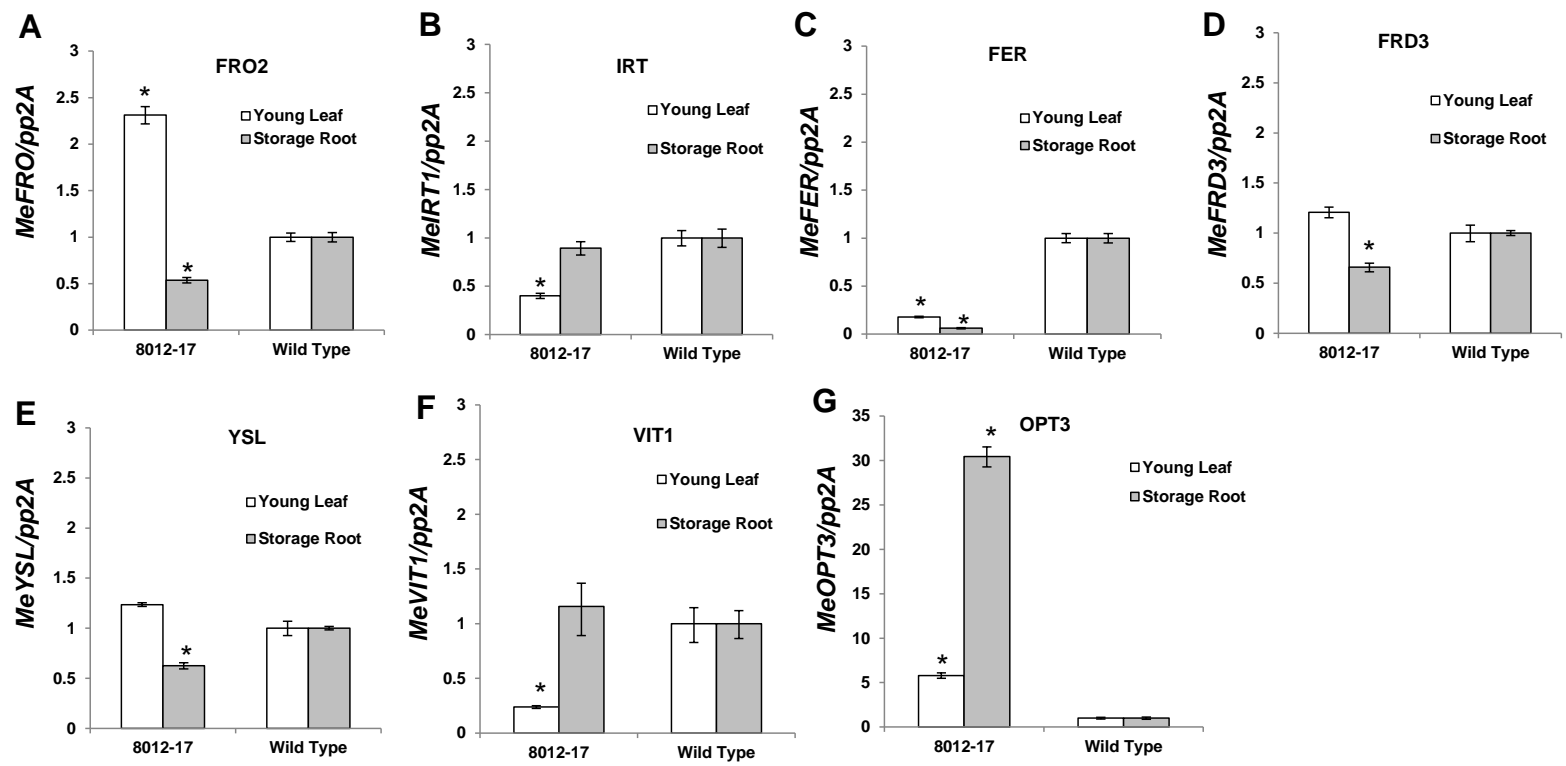

Figure 7 